\title{
Genetische Untersuchungen beim Kind
}

Medizinische, genetische, ethische und rechtliche Aspekte

Anlässlich der gemeinsamen Jahresversammlung der Schweizerischen Gesellschaft für Pädiatrie (SGP/SSP) und der Schweizerischen Gesellschaft für Medizinische Genetik (SGMG/SSGM) in Basel fand am 24. Juni 2004 ein Symposium mit obengenanntem Titel statt. Die vier Hauptreferate werden in diesem Heft zusammengefasst. In unserer Zeit mit zunehmenden genetischen Abklärungen wird eine breite Information über die Bedeutung von genetischen Untersuchen im Praxis- und Klinikalltag sowie über den Umgang mit den damit verbunden Problemen immer vordringlicher. Die genetische Analytik beim Kind und Jugendlichen hat besondere, sehr beachtenswerte Aspekte. Wir wünschen Ihnen eine anregende Lektüre!

Prof. Dr. med. Hansjakob Müller, Basel

\section{Genetische Untersuchungen bei Kindern und Jugendlichen}

\author{
Hj. Müller
}

Genetische Untersuchungen gewinnen in der Kinder- und Jugendmedizin an praktischer Bedeutung. Vielfach besteht noch Ungewissheit im Hinblick auf ihre Indikationen, ihre Aussagekraft oder auch ihre Vertretbarkeit gerade bei Personen, die die volle Autonomie noch nicht erreicht haben. In dieser Übersicht wird auf die Bedeutung und Realisierung genetischer Untersuchungen im Praxis- und Klinikalltag unter besonderer Berücksichtigung pädiatrischer Aspekte eingegangen.

\footnotetext{
* siehe auch Beitrag von Monica Gersbach-Forrer.
}

Korrespondenz:

Prof. Dr. med. Hansjakob Müller UKBB/DKBW

Abt. Medizinische Genetik

CH-4005 Basel

E-Mail:

hansjakob.mueller@unibas.ch
Es ist das erklärte Ziel der Schulmedizin - so auch der Pädiatrie -, der Ursache einer Krankheit oder Behinderung möglichst genau auf die Spur zu kommen, um darauf basierend die Behandlung auswählen oder Vorbeugemassnahmen ableiten zu können. Gerade diesem dienen genetische Untersuchungen, Gentests miteingeschlossen. Sie ersetzen immer häufiger aufwendige, für die Betroffenen gelegentlich recht unangenehme Diagnoseverfahren, wie z.B. solche, für die Gewebeproben benötigt werden.

\section{Indikationen für genetische Untersuchungen bei Kindern und Jugendlichen*}

Genetische Untersuchungen ermöglichen es, eine klinisch gestellte Verdachtsdiagnose zu bestätigen (Bestätigungsdiagnostik) oder zu verwerfen bzw. die klinische Diagnose zu präzisieren (z. B. Chromosomenanalyse bei Dysmorphologiesyndrom oder FraX-Gentest bei geistiger Behinderung).
Zunehmend wird die genetische Diagnostik zur Wahl und Dosierung von Medikamenten (RYR1-Gen bei Angehörigen aus Familien mit entsprechender Form der malignen Hyperthermie), für das Anpassen der Diät (PHA-Gen bei Phenylketonurie) bzw. für beides (OTC-Gen bei Mädchen mit Risiko, eine Konduktorin des X-gonosomal-vererbten Ornithintranskarbamylasemangels zu sein) benutzt.

Genetische Untersuchungen ermöglichen es, in von einer Erbkrankheit betroffenen Familien diejenigen Angehörigen, die die krankheitsverursachende Veranlagung geerbt haben, zu identifizieren, bevor klinische Symptome auftreten (RET-Onkogen beim MEN2-Syndrom ab dem 5. Lebensjahr; VHL-Gen bei der Von-HippelLindau-Erkrankung ab dem 5. Lebensjahr; APCGen bei familiärer adenomatöser Polyposis coli ab dem 10. Lebensjahr). Dank rechtzeitig getroffener Massnahmen lassen sich in der Folge Krankheitskomplikationen abwenden. Bei den nicht betroffenen Geschwistern erübrigen sich die eventuell aufwendigen medizinischen Betreuungsmassnahmen.

Schliesslich kann man auch genetische Eigenschaften bei Kindern oder Jugendlichen identifizieren, die für ihre eigene Gesundheit noch bedeutungslos sind (BRCA1/2-Mutation bei familiärem Vorkommen des Mamma-/Ovarialkarzinoms) oder die erst im Hinblick auf die Familienplanung (Verdacht auf Heterozygotie einer Mutation des CFTR-Gens oder möglicher Konduktorinnenstatus für die Muskeldystrophie 
Duchenne) relevant werden können. Von solchen Untersuchungen ist abzusehen (siehe unten)!

\section{Kinder und Jugendliche sind künftige Erwachsene mit eigener Autonomie**}

Das Respektieren der Autonomie der Patientin / des Patienten wurde Ende der 60er, Anfang der 70er Jahre zu einem Grundprinzip der medizinischen Ethik [1]. Diese zu achten und zu fördern ist ein wichtiges Anliegen der medizinischen Genetik. Mittels «informed consent» und nichtdirektiver Beratung versucht sie, sicherzustellen, dass die Werte der hilfesuchenden Personen und nicht diejenigen des Beraters Entscheidungen über den Umgang mit dem genetischen Schicksal bestimmen.

Bei der Veranlassung von genetischen Untersuchungen bei Kindern und Jugendlichen muss zwischen den Vorteilen des besseren Wissens um genetische Eigenschaften und möglichen ungünstigen Auswirkungen im Hinblick auf ihre Zukunft abgewogen werden. Kinder sind als Individuen zu respektieren, die dann mit 18 Jahren $\mathrm{zu}$ Erwachsenen werden. Man hat ihren Autonomieanspruch zu achten und dabei die zunehmende Entscheidungsfähigkeit zu berücksichtigen. Schon mit 7 Jahren sind sie in der Lage, an Entschlüssen teilzuhaben. Nach dem 12. Lebensjahr werden Konzepte wie Sterblichkeit, richtig und falsch, Zukunftsperspektiven oder Aufwand und Ergebnis nachvollziehbar. Die Mitbeteiligung der Kinder an Entscheidungsprozessen entbindet die Eltern jedoch nicht von ihrer Verantwortung für deren gesundheitliches Wohlergehen.

\section{Zum Spektrum der genetischen Untersuchungen}

Viele verstehen unter genetischen Untersuchungen nur solche, die mit gentechnischen Verfahren vorgenommen werden. Im neuen Bundesgesetz über genetische Untersuchungen beim Menschen (Art. 3) umfasst deren Definition die zytogenetischen und die molekulargenetischen Analysen zur Abklärung entweder ab Zeugung vorliegender oder während der Embryonalphase erworbener Eigenschaften des Erbguts sowie alle weiteren Laboruntersuchungen, die unmittelbar darauf abzielen, solche Informationen über das Erbgut zu gewinnen. Dabei handelt es sich vor allem um die Erfassung von zu Krankheit oder Behinderung führenden Veränderungen (Muta- tionen), d.h. um zahlenmässige und strukturelle Störungen der Chromosomen oder um Abweichungen derjenigen DNA-Abschnitte, die ein einzelnes proteinkodierendes Gen repräsentieren bzw. auch andere im Verlauf der Evolution konservierte Sequenzen.

\section{Genetischer Fortschritt dank technischer Errungenschaften}

Die Genetik fand in der Medizin früher kaum Beachtung. Einige Erbkrankheiten wie die Hämophilien A und B waren zwar bekannt; sie zählten aber zu den Raritäten. Technische Errungenschaften haben diese Situation verändert.

1952 erkannte T. C. Hsu nach zufälliger Verwendung einer hypotonischen Lösung zur Reinigung von Zellkulturen, dass man die wegen dieser Behandlung aufgequollenen mitotischen Zellen so präparieren kann, dass die einzelnen Chromosomen eines Zellkerns auf einem Objektträger nebeneinander zu liegen kommen und im Lichtmikroskop einzeln beurteilbar werden [2]. 1959 wurden die ersten Chromosomenstörungen beim Menschen nachgewiesen, so die Trisomie 21 beim Down-Syndrom durch J. Lejeune und Mitarbeiter [3], der 47,XXY-Chromosomensatz beim Klinefelter-Syndrom durch Patricia A. Jacobs und J. A. Strong [4] und das Fehlen eines Geschlechtschromosoms beim Turner-Syndrum durch C. E. Ford und Kollegen [5]. Mit der Entwicklung der Fluoreszenz-in-situHybridiserungs-(FISH-)Technik Ende der 80er Jahre können Verluste oder Duplikationen kleinster Chromosomensegmente sichtbar gemacht werden. Eine neue Gruppe von Erbkrankheiten wurde wahrgenommen, nämlich diejenige der sogenannten «Mikroaneuploidien».

Der Nachweis einer erhöhten Metachromasie der in vitro gezüchteten Fibroblasten von Patienten mit dem Morbus Hurler nach deren Anfärbung mit verschiedenen Farbstoffen durch B. S. Danes und A. G. Bearn im Jahre 1965 [6] stimulierte andere Wissenschafter, Zellkulturen zur Erforschung der Ursache und auch der Behandelbarkeit einer ganzen Reihe von Stoffwechselkrankheiten zu verwenden, so des Lesch-NyhanSyndroms, weiterer Mukopolysaccharidosen oder des Morbus Tay-Sachs.

Der entscheidende methodische Durchbruch in der Erforschung von Erbkrankheiten erfolgte am Anfang der 70er Jahre mit der Entwicklung der Gentechnologie oder «angewandten» molekularen Genetik [7]. Y. W. Kan und A. M. Dozy wiesen 1978 die Möglichkeit einer indirekten DNA-Diagnostik dank der Assozia- 
tion eines Schnittstellenpolymorphismus des Hpa-I-Restriktionsenzyms mit dem Sichelzellanämiegen nach [8]. 1982 gelang J. C. Chang und Y. W. Kan die pränatale Diagnostik der gleichen Krankheit, indem sie dafür die für die Sichelzellanämiemutation typische Elimination einer Schnittstelle des Mst-II-Restriktionsenzyms im Beta-Ketten-Hämoglobin-Gen für deren Nachweis nutzten [9]. Heute wird die DNA mit einem breiten Spektrum verschiedener gentechnischer Verfahren analysiert. Die auch für diagnostische Zwecke in Entwicklung begriffene Chiptechnologie verspricht ein breites Analysespektrum bei grossem und raschem Probendurchlauf.

DNA gibt es nicht nur im Zellkern [10]. Zwei bis zehn DNA-Moleküle kommen in jedem Mitochondrium vor. 1988 entdeckten Wallace und Mitarbeiter die erste mitochondriale DNA-Mutation, die zu Krankheit, nämlich zur Leber'schen Hereditären-Optikus-Neuropathie (LHON) führt [11].

Ohne die parallele rasante Entwicklung in der Datenverarbeitung/Informatik ( «research in silico») wäre die Bewältigung der heute anfallenden genetischen Daten und deren Interpretation im Hinblick auf mögliche klinische Folgen undenkbar.

\section{Zum aktuellen Stand des Genom- projektes}

Das in den 80er Jahren gestartete menschliche Genomprojekt hat zum Ziel, die molekulare Anatomie des gesamten Erbgutes des Menschen sowie dasjenige einiger Modellorganismen zu entziffern, also die Reihenfolge der Basensprossen der DNA-Doppelspirale festzuhalten und diejenigen DNA-Abschnitte zu erkennen, die Gene repräsentieren. Am 26. Juni 2000 feierte der damalige US-Präsident Bill Clinton in Begleitung von Craig Venter, dem Chef der privaten Sequenzierfirma Celera, sowie von Francis S. Collins, dem Repräsentanten des öffentlichen Humangenomprojektes, gemeinsam mit dem per Video zugeschalteten Premierminister Englands, Tony Blair, die Fertigstellung der Arbeitsversion des menschlichen Genoms [12]. Jedoch erst am 14. April 2003 erklärte dann das zuständige wissenschaftliche Konsortium den Abschluss des Genomprojektes, also die Abfolge der 3 Milliarden Basenpaare des menschlichen Erbgutes entziffert zu haben. Francis S. Collins bezeichnete das damals Erreichte «the end of the beginning».

Der aktuelle Stand des tatsächliches Wissens über den molekularen Aufbau unseres Erbgutes sowie über die darin enthaltenen Gene und deren medizinische Bedeutung kann der systematisch aufdatierten OMIM-Statistik entnommen werden (www.ncbi.nlm.nih.gov/Omim/ mimstats.html) (Tab. 1). Wenn man davon ausgeht, dass unser Erbgut 20000 bis 25000 Gene enthält [13], so wird bis heute erst etwa zwei Drittel davon erfasst. Nur bei einem kleinen Anteil kann aufgrund der gefundenen Mutationen verlässlich auf deren klinische Auswirkung geschlossen werden. Weltweit besteht nur für etwa 500 Gene ein Routinediagnostikangebot. Alle andern werden nur im Rahmen von Forschungsprojekten analysiert. Daher kann es sehr viel Überzeugungskunst und Zeit beanspruchen, damit eine Genuntersuchung von rein diagnostischer Bedeutung realisierbar wird, was leicht bei den Betroffenen, ihren Angehörigen, aber auch der sie betreuenden Ärzteschaft verständlicherweise Frustrationen auslöst. Sobald aber einmal in einer Familie bei einer Person die krankheitsverursachende Mutation erfasst ist, wird ihr Nachweis oder Ausschluss bei Angehörigen einfacher.

Tabelle 1

OMIM-Statistik vom 17. August 2004.

\begin{tabular}{|c|c|c|c|c|c|}
\hline & Autosomal & X-gonosomal & Y-gonosomal & Mitochondrial & Total \\
\hline Gene mit bekannter Sequenz & 9172 & 415 & 47 & 37 & 9671 \\
\hline $\begin{array}{l}\text { Gene mit bekannter Sequenz und } \\
\text { bekanntem Phänotyp }\end{array}$ & 352 & 36 & 0 & 0 & 388 \\
\hline $\begin{array}{l}\text { Phänotypbeschreibung und } \\
\text { molekulare Basis bekannt }\end{array}$ & 1447 & 134 & 1 & 24 & 1606 \\
\hline $\begin{array}{l}\text { Mendel'scher Phänotyp oder } \\
\text { Locus, molekulare Basis unbekannt }\end{array}$ & 1281 & 130 & 4 & 0 & 1415 \\
\hline $\begin{array}{l}\text { Andere Phänotypen mit } \\
\text { vermuteter Mendel'scher Basis }\end{array}$ & 2132 & 154 & 2 & 0 & 2288 \\
\hline Total & 14384 & 869 & 54 & 61 & 15368 \\
\hline
\end{tabular}




\section{Genetische Daten - genetische Informationen}

Wir Ärztinnen und Ärzte wurden während der letzten Jahrzehnte sehr laborgläubig erzogen. So trauen wir den Angaben in einem Laborbericht oft mehr als dem, was wir mit den eigenen Augen sehen. Wegen der Gleichsetzung des genetischen Codes mit unserer Schrift entsteht der Eindruck, dass bei der molekulargenetischen Analyse die Folge der Basenpaare so einfach wie Buchstaben hintereinander abgelesen werden. Dabei gehen aus Gentests vorerst oft recht abstrakte Ergebnisse hervor, die dann in schriftlich mitteilbare Laborresultate übergeführt werden müssen. Fehler können sich nicht nur bei der Auswertung der Rohdaten, sondern auch bei verschiedenen Schritten der Umsetzung der verschiedene Labormethoden einschleichen. Zudem lassen sich mit keiner einzelnen gentechnischen Methode alle Mutationsformen erkennen. So ist ein genetisches Laborresultat nicht einfach hinzunehmen, vor allem dann nicht, wenn es nicht mit dem Phänotyp übereinstimmt. Diese kritischen Anmerkungen wollen aber keineswegs besagen, dass sich mit genetischen Untersuchungen nicht sehr verlässliche Resultate erzielen lassen.

Der Mensch und so auch ein Kind ist mehr als ein multizelluläres, durch seine Gene gesteuertes Lebewesen. Es will als Individuum mit seiner eigenen Geschichte und in seiner Umwelt wahrgenommen werden. In diesem Gesamtkontext sind auch genetische Daten zu deuten und in für den Probanden relevante Informationen überzuführen. Dies erfordert neben medizinischem und genetischem Fachwissen klinische Erfahrung, Einfühlungsvermögen und Umsicht. Es wird befürchtet, dass bei der immer effizienter werdenden Produktion genetischer Daten die Sorgfalt bei ihrer Auslegung leiden könnte und diesbezüglich gefährliche Automatismen aufkommen könnten.

\section{Ausblick in eine Zukunft mit mehr Gentests}

Beim raschen Fortschritt der genetischen Forschung gelingt es nicht mehr, in Lehr- und Sachbüchern den aktuellen Wissensstand über Gentests oder die für die Interpretation der daraus hervorgehenden Resultate benötigten Informationen vollumfänglich wiederzugeben. Somit ist man häufig auf über Internet abrufbare Informationsquellen angewiesen. OMIM (www3.ncbi. nlm.nih.gov.omim) ist die Wissensressource über menschliche Gene, deren bekannte Muta- tionen und klinischen Auswirkungen. GeneTests/GeneClinics (www.genetests.org) und Orphanet (www.orpha.net) geben gute verständliche Informationen über die verschiedenen Erbkrankheiten bzw. über deren molekulargenetischen Nachweis wieder.

\section{Genetik und Medizin in der Schweiz}

Die Genetik bedarf unbestreitbar der Förderung im schweizerischen Medizinalwesen. Die in weiten Kreisen der Ärzteschaft, überhaupt des Medizinalpersonals, ihr gegenüber spürbare $\mathrm{Zu}$ rückhaltung ist unter anderem die Folge eines während vieler Jahre ungenügenden Aus-, Weiter- und Fortbildungsangebotes.

Das gesamte Umfeld der genetischen Untersuchungen wird immer komplexer. Das Überführen genetischer Daten in für die untersuchte Person relevante genetische Informationen ist anspruchsvoll. Die Beurteilung des Angebotes von Präventions- und Therapiemassnahmen erfordert fächerübergreifende Weitsicht. Eine vermehrte, gut eingespielte Zusammenarbeit aller beteiligter Medizinalpersonen wird bei der genetischen Diagnostik und beim Ableiten der aus einem Ergebnis angezeigten Konsequenzen immer vordringlicher. Diese ist bei uns noch schlecht eingespielt, so dass Patientinnen/ Patienten mit genetischen Problemen oft einer Odyssee zwischen all den medizinischen Fachleuten mit der ihnen eigenen Sicht und Sprache ausgesetzt sind. Dadurch verursachte, unnötige Verunsicherungen wirken sich bei Kindern und Jugendlichen sowie fürsorglichen Eltern besonders ungünstig aus.

\section{Literatur}

1 Beauchamp TL, Childress JF. Principles of Biomedical Ethics. $4^{\text {th }}$ edition. Oxford: Oxford University Press; 1994.

2 Hsu TC. Human and Mammalian Cytogenetics. An Historical Perspective. New York, Heidelberg, Berlin: Springer; 1979.

3 Lejeune J, Gauthier M, Turpin R. Étude des chromosomes somatiques de neuf enfants mongoliens. C R Acad Sci. 1959;248:1721-2.

4 Jacobs PA, Strong JA. A case of human intersexuality having a possible XXY sex-determining mechanism. Nature 1959;183:302-3.

5 Ford CE, Miller OJ, Polani PE, de Almeida JC, Briggs JH. A sex-chromosome anomaly in the case of gonadal dysgenesis (Turner's syndrome). Lancet 1959;1:711-3.

6 Danes BS, Bearn AG. Hurler's syndrome: a genetic study in cell culture. J Exp Med 1966;125:1-7. 
7 Watson JD, Tooze J. The DNA Story. San Francisco: Freeman \& Co.; 1981.

8 Kan YW, Dozy AM. Polymorphism of DNA sequence adjacent to human beta-globin structural gene: relationship to sickle mutation. Proc Natl Acad Sci U S A 1978;75(11):5631-5.

9 Chang JC, Kan YW. A sensitive test for prenatal diagnosis of sickle cell anemia: direct analysis of amniocyte DNA with MstII. Trans Assoc Am Physicians 1982;95:71-8.

10 Schatz G, Haselbrunner F, Tuppy H. Desoxyribonucleic acid associated with yeast mitochondria. Biochem Biophys Res Commun 1964;15:127-32.
11 Wallace DC, Singh G, Lott MT, Hodge JA, Schurr TG, Lezza AM, et al. Mitochondrial DNA mutation associated with Leber's hereditary optic neuropathy. Science 1988;242:1427-30.

12 Macilwain C. World leaders hap praise on human genome landmark. Nature 2000;405:983-4.

13 International Human Genome Sequencing Consortium. Finishing the euchromatic sequence of the human genome. Nature 2004;431:931-45.

\title{
Tests génétiques chez les enfants et les adolescents
}

\author{
M. Gersbach-Forrer
}

Dans un sens large, la génétique peut être définie comme la science de la variation et de la ressemblance. D'une génération à la suivante, une part de soi est transmise, une autre ne l'est pas, la part transmise comporte $50 \%$ de notre bagage génétique mais sa composition n'est jamais la même. Chaque descendant aura donc un bagage génétique unique comportant des ressemblances mais aussi des variations par rapport à ses parents. Cette constellation génétique apparentée mais aussi différente fait que chaque enfant, chaque parent connaît probablement des instants où l'idée de famille réjouit ... et d'autres où quelques tailles et boutures de l'arbre généalogique seraient les bienvenues.

Correspondance:

Dr Monica Gersbach-Forrer Centre médical universitaire Service de génétique médicale Rue Michel-Servet 1 CH-1211 Genève 4

E-mail:

monica.gersbach@medcli.unige.ch

\section{En quoi les tests génétiques sont-ils particuliers, voire le sont-ils vraiment?}

\section{Un test pour la vie, «non retour»}

Contrairement à de nombreuses analyses médicales, un test génétique a un seul et même résultat au cours de toute la vie, qu'il soit fait avant la naissance, dans l'enfance, chez un adulte, avant ou après la survenue de symptômes cliniques. Une analyse du groupe sanguin ou une typisation HLA présentent ces mêmes caractéristiques et peuvent être considérées comme des tests génétiques au sens large.

\section{Implications/risques pour les apparentés}

Un test génétique peut révéler un risque augmenté pour d'autres apparentés, parfois révéler leur status relatif à une affection familiale, de plus, il s'agit souvent d'un test pour une affec- tion touchant déjà certains membres de la famille. Dans ce sens, même si chaque test est géré dans la confidentialité, il est souvent difficile de faire abstraction de l'expérience, du vécu, d'attentes, de règles non écrites (par exemple de solidarité, de loyauté) au niveau de l'histoire familiale. Même dans la confidentialité optimale, on n'est souvent pas tout à fait seul et indépendant face à un test génétique.

\section{Confidentialité}

De même que dans certains autres types d'analyses (HIV par exemple), la confidentialité est une priorité absolue dans le cadre de tout test génétique. Il y a cependant des situations où des apparentés doivent être avertis d'un risque important les concernant. Ce fait pose éventuellement une limite à la confidentialité dans une pesée d'intérêt entre des exigences divergentes.

\section{Choix de vie, décisions importantes}

Une étude récente sur environ 350 consultations de génétique médicale dans notre service a montré que plus de la moitié de nos consultations implique une décision jugée importante par la/les personne(s) qui consulte(nt). Les décisions pour ou contre la mise en route ou la poursuite d'une grossesse, pour ou contre un test génétique surtout s'il est prénatal ou présymptomatique posent des questions de choix de vie avec lesquelles une personne, un couple doit pouvoir 
vivre à long terme. Pour un couple qui souhaite un diagnostic prénatal pour une affection familiale par exemple, il est fortement recommandé (si possible) de l'adresser en génétique médicale avant le début d'une grossesse afin d'assurer une information et une décision dans des conditions optimales. De plus, il n'est pas rare qu'un diagnostic prénatal nécessite une préparation technique ou qu'une collaboration avec une autre équipe travaillant sur un gène particulier doive être mise en place au préalable. De longs mois sont donc parfois nécessaires avant qu'un diagnostic prénatal fiable puisse être proposé.

\section{Implications éthiques, quelles valeurs humaines?}

Les tests génétiques posent souvent des questions éthiques importantes qui doivent être abordées en profondeur avec les personnes qui consultent et ne réalisent pas forcément d'emblée la portée à court et à long terme des décisions qu'elles vont être amenées à prendre. Il est ainsi souhaitable que les options envisagées soient abordées en détail, parfois en plusieurs séances et en aménageant un temps de réflexion afin qu'un choix réellement éclairé puisse avoir lieu. Ce n'est pas parce que l'on peut techniquement faire un test que celui-ci est forcément le bon choix pour la personne concernée. Il est frappant de constater que pour le diagnostic présymptomatique de la chorée de Huntington, plus de $3 / 4$ des personnes directement à risque (risque de 50\% de développer la maladie ultérieurement) décide de renoncer à un test et choisit par conséquent souvent de mettre au monde des enfants avec un risque de $25 \%$ de développer l'affection familiale. On imagine aisément qu'une telle décision n'est pas simple à prendre au niveau d'un couple et nécessite un contact sur un certain temps avec un médecin généticien compétent dans un domaine très spécialisé de la psychologie médicale.

\section{Autres éléments importants}

D'autres éléments importants interviennent aussi dans l'appréciation d'un test génétique qui ne peuvent pas être abordés plus en profondeur ici, par exemple: la valeur informative du test, le contrôle de qualité du laboratoire, les difficultés liées à l'interprétation du résultat, le risque potentiel de résultat imprévu, de révéler une nonpaternité. Il est primordial que les laboratoires soient sensibles aux problèmes spécifiques posés par les tests génétiques afin que leur réponse soit appropriée et les abus évités.

\section{Loi fédérale sur les tests génétiques}

Le projet avancé de loi fédérale sur les tests génétiques chez l'humain apportera un cadre très attendu dans la pratique de la génétique médicale.

Tous ces éléments doivent être abordés minutieusement afin d'assurer un choix réellement éclairé, condition sine qua non d'un consentement éclairé, d'où l'importance d'un conseil génétique par une personne qualifiée avant tout test génétique.

On distingue schématiquement deux niveaux de tests génétiques, les approches techniques sont différentes avec cependant une certaine convergence depuis l'introduction de l'hybridisation fluorescente in situ (FISH).

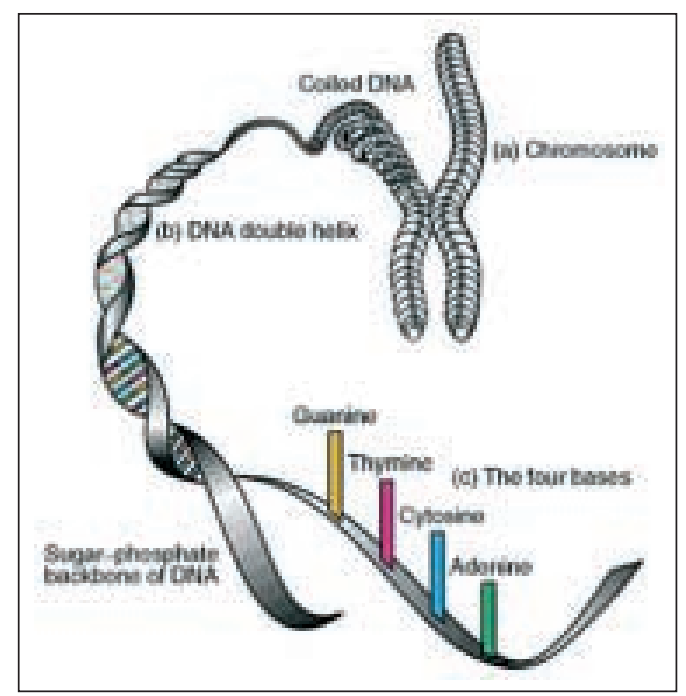

Le caryotype va donner une image photographique des chromosomes permettant d'identifier des changements de nombre et de structure dans la limite du niveau de résolution obtenu.

Les analyses $A D N$ où les outils de la biologie moléculaire permettent d'identifier un changement (une mutation) au niveau d'un ou de plusieurs gènes, c'est-à-dire au niveau de la séquence d'ADN.

La méthode FISH permet de visualiser sur un caryotype la présence ou l'absence d'un signal fluorescent lié à une région génique précise.

La majorité des tests génétiques chez l'enfant ont un but diagnostique dont l'intérêt immédiat pour la prise en charge prime sur les autres implications potentielles (familiales, confidentielles, etc.).

Certains tests génétiques peuvent être envisagés chez un enfant sain: lesquels? pourquoi? quand? 
Types de tests génétiques parfois envisagés chez un enfant sain:

\section{Analyses chromosomiques}

- Translocations: anomalie de structure («équilibrée» chez les porteurs sains avec risque de déséquilibre pour la descendance);

- anomalie du nombre de chromosomes sexuels (p.ex. XXY syndrome de Klinefelter: infertilité).

\section{Analyses d'un gène responsable d'une} affection suivant le mode d'hérédité

- Autosomique-récessif: une personne chez laquelle les deux gènes de la paire en cause sont modifiés (II) est atteinte, les parents (si non atteints) sont des porteurs sains obligatoires avec un gène modifié et un gène fonctionnel (II), ce dernier assurant une fonction suffisante (p.ex. gène CFTR de la mucoviscidose (cystic fibrosis) ou nombreux gènes responsables d'affections métaboliques, etc.).

- Autosomique-dominant où il suffit qu'un gène de la paire soit modifié pour que la personne soit affectée (II):

- pathologies à début tardif: test génétique présymptomatique;

- syndromes de prédisposition à certains risques (cancer, Marfan, etc.).

- Lié à l'X où les hommes qui portent un X modifié sont atteints (XY) alors que les femmes sont des conductrices saines $(\mathrm{XX})$, le gène situé sur le chromosome $\mathrm{X}$ intact assurant une fonction suffisante (Hémophilie, myopathie de Duchenne, syndrome de l' $X$ fragile, etc.).

\section{Exemple de quatre situations où la question d'un test génétique chez l'enfant est posée}

\section{Une translocation chromosomique chez un parent}

Un couple avec deux enfants en bonne santé où l'anamnèse de fausses couches à répétition ( $\geq 3$ ) pose l'indication pour un caryotype chez les deux partenaires.

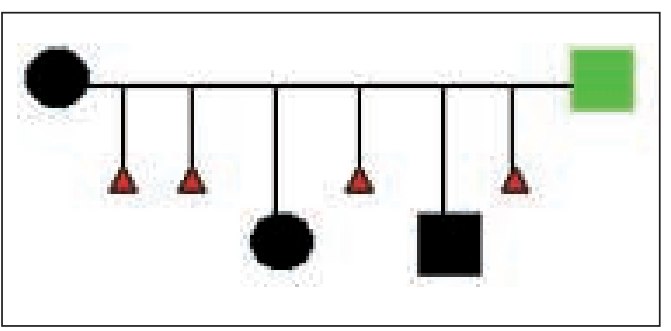

A l'analyse chromosomique, on détecte chez Monsieur une anomalie de structure et de nombre: une translocation 13/14 balancée (équilibrée), c'est-à-dire 45 chromosomes au lieu de 46 avec un chromosome très long formé par un chromosome 13 accolé à un 14. En dehors du problème reproductif, le père n'a aucun inconvénient lié à cette particularité où il n'y a en principe ni perte ni adjonction de matériel génétique exprimé.

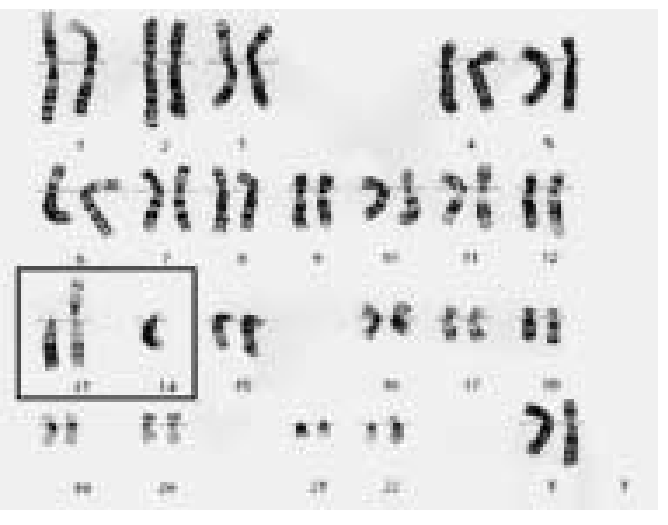

Le couple demande une carte chromosomique chez leur deux enfants qui ne présentent aucun problème de santé.

Théoriquement, la descendance de ce couple peut présenter:

- un caryotype normal (chromosomes 13 et 14 normaux du père transmis);

- un caryotype avec la translocation équilibrée 13/14 comme le père;

- une trisomie 13 (syndrome de Patau) si le chromosome transloqué (13/14) est transmis avec en plus un chromosome 13 normal du père;

- une trisomie 14 si le chromosome transloqué est transmis avec un chromosome 14 normal. Dans ce cas, la grossesse est non viable et va évoluer en fausse couche précoce;

- une monosomie 13 si ni le chromosome transloqué ni le 13 libre n'ont été transmis par le père, la grossesse est précocement non viable;

- une monosomie 14 si ni le chromosome 14 transloqué ni le 14 libre du père n'ont été transmis. La grossesse va rapidement évoluer en fausse couche.

La littérature et l'expérience montrent que dans ce cas, ce sont de très loin les deux premières possibilités qui surviennent le plus fréquemment alors que les 4 possibilités de déséquilibre chromosomique ne surviennent que très rarement (quelques pour cent) et ont un haut risque de fausse couche. Le risque de trisomie 13 est faible, 
mais justifie de proposer un diagnostic prénatal. Les fillettes du couple étant saines, elles peuvent présenter une carte chromosomique normale ou avec la translocation équilibrée comme leur père.

\section{Tester ou non? Pourquoi, pourquoi pas?}

Il s'agit d'un test de «porteur sain» sans implications avant l'âge reproductif. Un certain nombre d'éléments doivent être pris en compte. Les parents peuvent faire valoir:

- leur droit de savoir;

- leur souffrance personnelle, leur vécu de couple avec perte répétée de grossesse;

- ils veulent «le bien de l'enfant»;

- ils sont responsables de l'enfant;

- ils sont deux, ils ne sont pas forcément ensemble, pas toujours du même avis.

En ce qui concerne les enfants, ces éléments sont:

- le respect de leur autonomie: le test abroge leur droit de décision de futur adulte;

- un test lèse leur droit de futur adulte (et celui de leur futur couple) à la confidentialité;

- leur compétence actuelle à participer à la décision;

- leur compétence actuelle à donner un consentement valable;

- le risque de stigmatisation, d'un regard changé sur un enfant porteur sain.

2. Une mutation dans le gène codant pour la protéine «dystrophine» et situé sur le bras court du chromosome $X$ (locus Xp21.2)

Cette mutation est responsable de

- la dystrophie musculaire de Duchenne (lorsque la mutation cause une altération complète de la fonction protéique);

- la dystrophie musculaire de Becker, moins sévère car la fonction du gène est partiellement conservée.

Une mutation du type Duchenne est identifiée chez l'aîné d'une fratrie de quatre suite à des chutes répétées et une peine progressive à monter les marches d'escaliers.

La maladie de Duchenne entraîne une faiblesse musculaire proximale progressive, des complications respiratoires et orthopédiques ainsi qu'une cardiomyopathie. L'enfant devient dépendant d'une chaise roulante vers l'âge de 12 ans, l'espérance de vie reste fortement diminuée à ce jour. Ce diagnostic implique une hérédité monogénique liée au sexe puisque le gène est situé sur le chromosome $\mathrm{X}$ : le sexe mâle est atteint XY; le sexe féminin est porteur sain XX, le second gène situé sur l'autre chromosome $\mathrm{X}$ étant normal et assurant une fonction suffisante.
Figure 4

Analyse $A D N$ d'un gène situé sur le chromosome $X$.
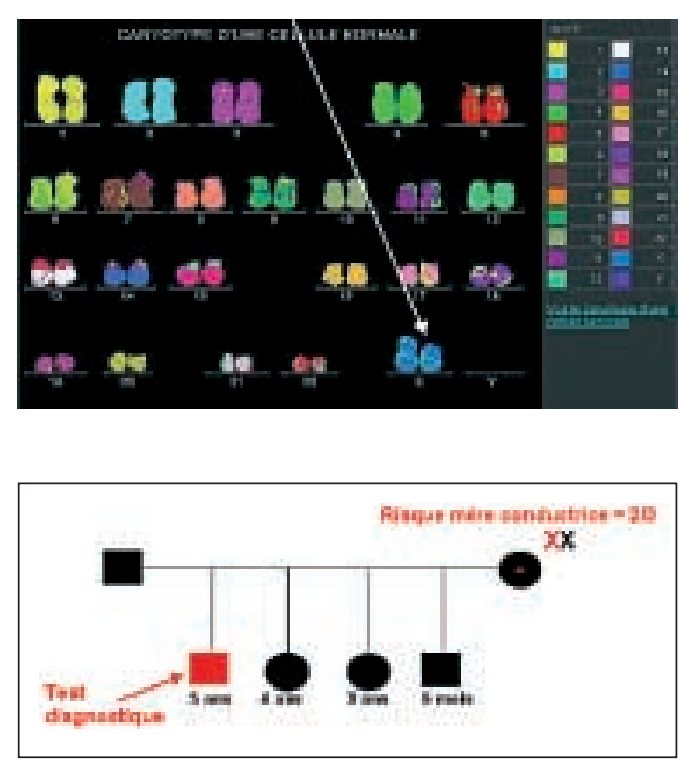

Lorsqu'il s'agit d'un cas sporadique, la probabilité que la mère soit porteuse saine est de $2 / 3$.

Une fois le choc du diagnostic un peu atténué et dans le contexte d'un conseil génétique où les aspects psychologiques ont une large part, un test génétique chez la mère a montré qu'elle est porteuse saine (ou conductrice) de la même mutation que son fils.

Par la suite, le couple demande une recherche de mutation chez leurs trois enfants sains:

- leurs filles ont un risque de $50 \%$ de porter la mutation: test de conductrice (= porteuse saine);

- leur fils cadet a un risque de 50 \% de développer la maladie: test prédictif pour une affection sévère sans traitement curatif à début dans l'enfance.

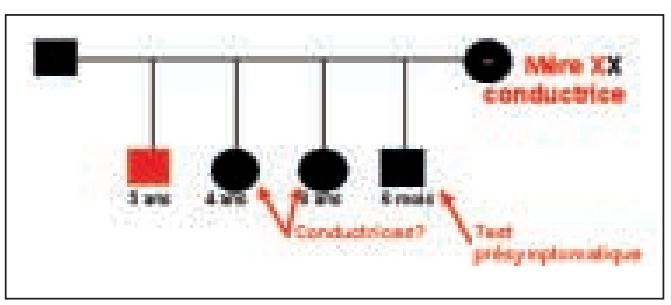

Un test de conductrice chez les sœurs implique

- l'abrogation d'une prise de décision autonome à l'âge adulte;

- l'abrogation de leur droit à une confidentialité individuelle et de couple une fois adultes tout particulièrement concernant les décisions relatives au planning familial et au choix éventuel d'un diagnostic prénatal face aux parents et au frère atteint. 
Chez le frère cadet, un test prédictif d'une affection sévère, incurable, débutant dans I'enfance pose des questions sans réponse simple

- Les parents ont-ils accusé le coup du diagnostic chez leur aîné?

- Peuvent-ils déjà supporter un potentiel second choc? L'incertitude est-elle plus lourde à supporter qu'une certitude éventuellement très douloureuse?

- Le test pourrait permettre aux parents de se préparer.

- Au niveau de la prise en charge, il n'y a aucune urgence à tester le fils cadet.

3. Une mutation dans un gène impliqué dans un syndrome de prédisposition à certains cancers: l'exemple du cancer du côlon (CC)

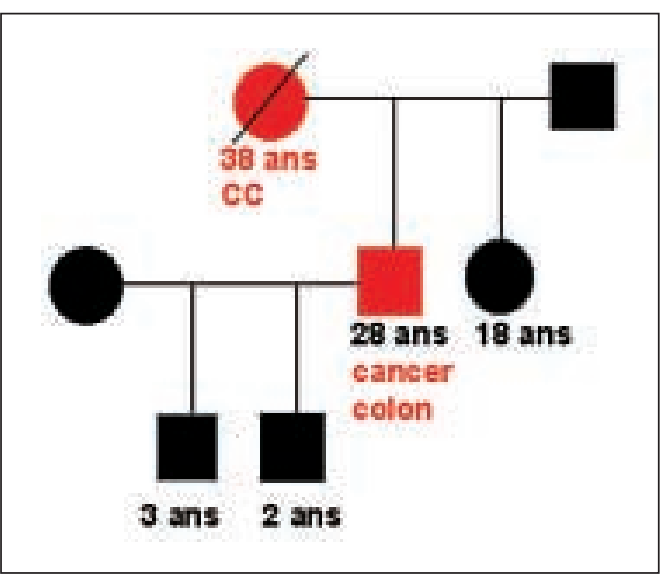

Dans cet exemple, un cancer du côlon est diagnostiqué chez un patient de 28 ans, inhabituellement jeune pour cette affection, et dont la mère est décédée précocement, anamnestiquement d'une affection similaire.

D’une façon générale, les syndromes génétiques de prédisposition à certains types de cancer dépendent d'une mutation dans un gène. La majorité des gènes de ce type actuellement connus ont un mode d'hérédité autosomique dominant (mais il en existe qui sont soumis au mode récessif). Le mode autosomique dominant implique un risque de $50 \%$ pour les apparentés du $1^{\text {er }}$ degré, de $25 \%$ pour ceux du $2^{\text {e }}$ degré (etc.) de présenter la même prédisposition.

A l'heure actuelle, un certain nombre de critères cliniques ont été définis permettant d'identifier les personnes atteintes (le probant) chez lesquelles une analyse génétique doit être proposée.

Lorsqu'une mutation est identifiée chez un probant, le test génétique va permettre:
- de proposer un test aux apparentés à risque qui le souhaitent afin d'identifier les porteurs de la mutations et d'instaurer chez eux des coloscopies régulières et une surveillance des autres organes potentiellement à risque, parfois une chirurgie prophylactique;

- de libérer de la surveillance les apparentés ne portant pas la mutation dont le risque est celui de la population générale.

On distingue à l'heure actuelle principalement (il en existe d'autres) deux syndromes de prédisposition au cancer du côlon:

\section{Le syndrome de Lynch = HNPCC Hereditary} Nonpolyposis Colorectal Cancer

- Certains critères cliniques (critères d'Amsterdam, de Bethesda) tels que jeune âge, tumeurs synchrones, métachrones, instabilité des microsatellites, immunohistochimie altérée, etc. donnent une indication à une analyse ADN ciblée.

- Les gènes impliqués appartiennent au groupe MMR (DNA missmatch repair genes, plusieurs gènes connus à ce jour).

- Le cancer se développe sur des polypes isolés et au niveau de certains autres organes (endomètre, estomac, ovaires, pancréas, système urinaire, etc.).

- Le risque au cours de la vie est élevé: de l'ordre de $75 \%$ pour le cancer du côlon, de $40 \%$ pour l'endomètre.

- Une surveillance ciblée est indiquée dès l'âge de 20 ans.

\section{La polypose adénomateuse familiale (FAP)}

- Le cancer se développe sur une polypose massive apparaissant dès l'âge de 7 ans et généralement avant l'âge de 36 ans.

- Dans 95\% des cas une mutation dans le gène APC peut être identifiée.

- Une mutation dans ce gène implique un risque avoisinant $100 \%$ de cancer du côlon au cours de la vie avec un âge moyen d'apparition de 39 ans.

- Ce risque est augmenté précocement, 7\% déjà à l'âge de 21 ans.

- Une surveillance ciblée est indiquée dès l'enfance au niveau du côlon, une chirurgie prophylactique peut être envisagée. Le risque d'hépatoblastome est augmenté dans l'enfance.

Dans l'exemple choisi, le bilan va dépendre du type de prédisposition en cause. 


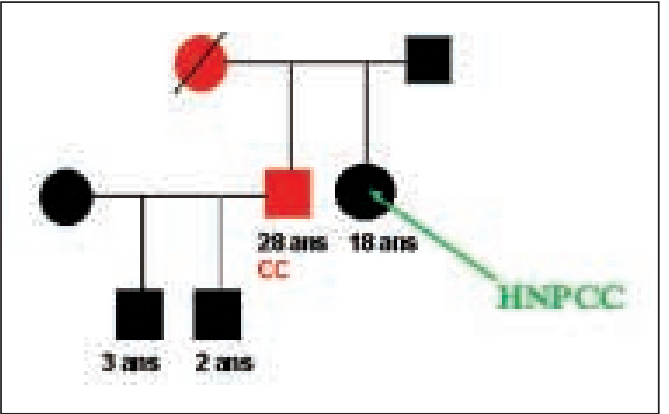

En cas de syndrome de Lynch (HNPCC), seule la sœur du probant est proche de l'âge où le risque est augmenté et un conseil génétique autour de l'indication à un test génétique doit lui être proposé. Les parents des deux jeunes enfants sont informés de l'importance du conseil génétique chez leurs enfants dès leur majorité.

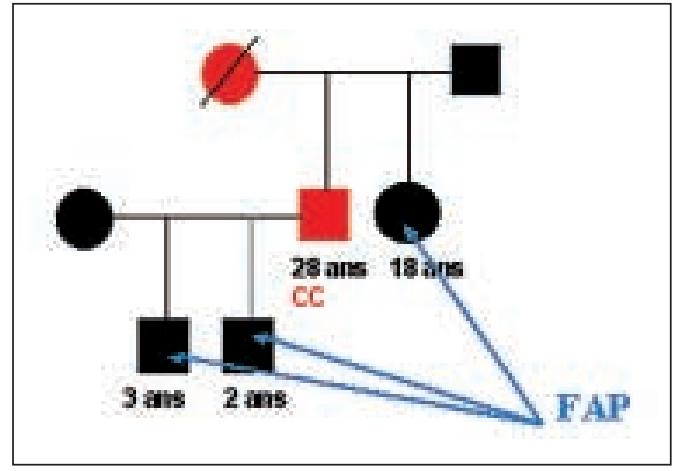

$\mathrm{Au}$ contraire, en cas de polypose familiale (FAP), il est justifié de proposer un test aussi chez les enfants mineurs du patient vu le risque augmenté déjà dans l'enfance en cas de mutation APC.

Il est aisé d'imaginer comment un test ADN, techniquement simple une fois la mutation familiale identifiée, lié à de tels risques peut interférer avec les projets de planning familial des couples concernés. A partir du moment où un test génétique peut être indiqué déjà dans l'enfance, certains couples vont être tentés de demander si ce test peut déjà se faire en prénatal. Généralement en effet, ceci ne poserait pas de problèmes au niveau strictement technique mais en pose de majeurs au niveau éthique pour une affection où une surveillance ciblée permet de réduire significativement un risque admis comme considérable. Les équipes onco-génétiques en charge du conseil génétique de tels couples sont très réticentes envers ce type de demandes actuellement très rares. Il n'existe pas de réponse simple à une telle demande. On peut imaginer qu'une équipe clinique souhaite ne pas entrer en matière pour des raisons éthiques, mais il semble alors éthiquement tout aussi important d'informer clairement que la demande peut être adressée et acceptée ailleurs. Il est intéressant d'observer que le fait d'exposer et de discuter les implications éthiques en profondeur (ce qui est essentiel dans chaque cas) remet très souvent en cause la demande initiale. Il est actuellement très rare qu'un couple confirme sa demande (aucun cas dans notre expérience à Genève). Il convient cependant de se préparer à un avenir qui pourrait être différent et être préparé à une telle demande faite dans le contexte d'un vécu particulièrement douloureux.

\section{Exemple d'une affection monogénique à début tardif: la maladie de Huntington (Huntington's disease, HD)}

Il s'agit d'un couple séparé depuis 10 ans avec deux enfants adultes. L'épouse, atteinte de sévères troubles de la personnalité est retournée dans son pays d'origine où elle vit en institution psychiatrique, elle est actuellement âgée de 42 ans. La fille du couple âgée de 23 ans a une fillette en bonne santé tandis que la partenaire du fils est enceinte depuis peu.

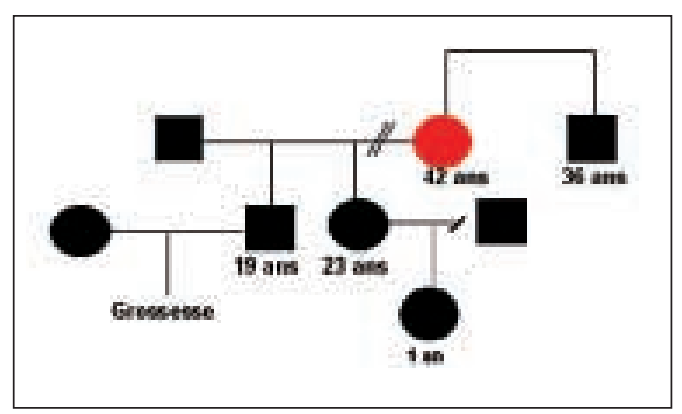

Pour rappel, il s'agit d'une affection neuro-dégénérative sévère associant des symptômes neurologiques à une perte cognitive et à une atteinte d'ordre psychiatrique. Elle débute à l'âge adulte, généralement autour de la quarantaine. L'hérédité est autosomique-dominante. L'analyse moléculaire de l'unique gène associé et situé sur le bras court du chromosome 4 , permet de confirmer un tableau clinique suggestif. La mutation en cause est d'un type particulier, elle est constituée d'une séquence de triplets ( 3 bases: CAG) répétés un certain nombre de fois. La taille de la mutation est instable et tend à augmenter en longueur d'une génération à la suivante, on parle d'une mutation «dynamique». Le nombre de répétitions présente une certaine corrélation avec l'âge auquel débute la maladie: plus le nombre de répétitions est grand plus l'affection risque de débuter précocement. Ceci explique pourquoi la maladie tend à débuter plus précocement d'une génération à la suivante avec, exceptionnellement, des cas débutant dans l'enfance ou l'adolescence. 
Dans une famille affectée, ce test, d'une très haute fiabilité, peut également être proposé avant tout signe clinique chez les apparentés à risque. Toute personne apparentée du $1^{\mathrm{er}}$ degré a un risque de $50 \%$ de développer la maladie, de $25 \%$ si elle est apparentée au $2^{\mathrm{e}}$ degré. Le même test peut aussi être proposé en prénatal.

S'agissant de la famille citée en exemple, on imagine aisément l'ampleur des répercussions d'un tel diagnostic chez cette femme de 42 ans. L'époux séparé réalise que ses problèmes de couple ont potentiellement été liés aux prémices d'une affection sévère et en même temps que ses enfants risquent de la développer à leur tour et, le cas échéant, de la transmettre aussi. Il en va de même pour les enfants et leurs partenaires.

Dans un tel contexte, il convient de procéder par étapes. Initialement, bien des familles souhaitent un entretien de conseil génétique commun. Dans un deuxième temps et au moment où cela leur convient, chaque personne concernée est revue pour un conseil génétique en plusieurs étapes incluant un temps de réflexion afin d'assurer un choix éclairé face aux possibilités de tests ou à l'option d'y renoncer. La présence d'un accompagnant proche, ami, partenaire est fortement recommandée; au besoin, le médecin généticien va s'entourer d'un collègue psychiatre et/ou neurologue. Dans ce contexte se pose souvent la question de savoir quand commence «l'âge adulte» pour de telles décisions et comment évaluer si un consentement réellement éclairé peut être donné. D’une manière générale, un test génétique présymptomatique pour une affection de cette gravité est vivement déconseillé avant l'âge adulte et la plus grande précaution doit être de mise chez toute personne qui en fait la demande. Un conseil génétique en profondeur, en plusieurs séances, comprenant une exploration des options possibles et de leurs conséquences nécessite beaucoup de temps et de disponibilité, il est une condition sine qua non pour un choix réellement éclairé et autonome face à un test présymptomatique ou prénatal, voire une interruption thérapeutique de grossesse.

Il existe dans le cas de la maladie de Huntington des recommandations sur l'utilisation des moyens de diagnostic présymptomatique issues

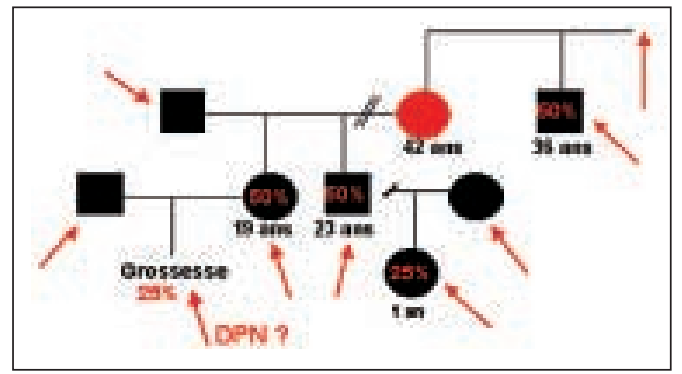

de l'Association Huntington international (AHI) et de la Fédération mondiale de neurologie (FMN).

Un tel conseil génétique extensif va être proposé à toutes les personnes à risque augmenté et/ou concernées directement: le conjoint, les enfants du patient, leur partenaire, les frères et sœurs du patient et le cas échéant des apparentés plus éloignés à risque. Dans le cas d'un enfant mineur, ces consultations vont aussi aborder en détail les raisons éthiques pour lesquelles un test génétique ne peut être fait chez un enfant même à la demande pressante de ses parents alors que l'on pourra accéder à une demande de diagnostic prénatal (DPN).

Le conseil génétique doit prendre en compte la représentation et le vécu de la maladie dans une famille donnée, apporter une connaissance réaliste de la maladie et des risques associés. Outre la discussion sur la manière dont la personne souhaite gérer les risques, il est primordial d'aborder d'autres domaines sensibles, celui des émotions et des moyens pouvant aider à les gérer, celui de la confidentialité et des problèmes potentiels à ce niveau (en particulier dans la relation à l'employeur) ainsi que celui relatif aux assurances privées (maladie, vie, etc.).

\section{En guise de conclusion}

On peut dire qu'il existe un large consensus parmi les professionnels en génétique humaine sur les points suivants:

Les tests génétiques chez les mineurs ne sont admissibles qu'en cas d'implications immédiates pour leur prise en charge au niveau du traitement, de la prévention et/ou de la surveillance.

Les tests présymptomatiques chez les enfants: - pour une affection débutant dans l'enfance: un tel test peut permettre aux parents de se préparer au futur;

- pour une affection débutant à l'âge adulte: réticence absolue vu l'abrogation d'une prise de décision autonome à l'âge adulte ainsi que du droit du futur adulte à une confidentialité individuelle et de couple incluant tout particulièrement les décisions relatives au planning familial.

Tests de porteurs sains chez les enfants: fortement contre-indiqués par les professionnels, très controversés parmi les associations de personnes concernées dont une proportion non négligeable estime que les parents ont un droit légitime de savoir et que ce fait ne nuit pas aux enfants concernés.

Les principaux intérêts en jeu sont donc: 


\section{Les droits actuels et futurs de l'enfant}

- défense de son «autonomie» et de son «droit de ne pas savoir» de futur adulte;

- protection de la sphère privée du futur adulte;

- protection du risque de stigmatisation, discrimination.

\section{Les droits des parents}

- droit de savoir, incertitude = angoisse, «désir d'échapper»;

- responsabilité de défendre au mieux les intérêts de l'enfant.

Lorsqu'un test génétique est envisagé chez un enfant ou un adolescent sain, les questions suivants devraient être abordées et/ou prises en compte dans le conseil génétique:

- Comment juger de la capacité d'un enfant ou d'un adolescent à donner un consentement «éclairé», à participer à une décision?

- Quel est l'âge où l'on devient autonome pour une telle décision? Cerner le moment à partir duquel un enfant donné est devenu un jeune adulte capable d'autonomie pour des choix difficiles peut être un sérieux casse-tête autant pour les parents que pour le médecin.

- Peut-on espérer des progrès thérapeutiques d'ici que le jeune soit adulte?

- Un refus rigide des professionnels est contreproductif.

- Une analyse en profondeur des causes et de la stabilité de la demande parentale ou d'un jeune est primordiale: Le test va-t-il améliorer la situation, l'exacerber, la demande est-elle éventuellement l'expression d'un problème plus complexe et profond qu'il convient d'aborder?

- Chaque cas est et doit rester un cas unique!
Bien avant un test, la grande question est souvent «Comment et quand parler d'un problème génétique grave avec ses enfants?». Ce sujet difficile fait partie du dialogue dans la durée entre parents, médecin traitant et professionnels en génétique médicale.

Les parents sont déchirés entre:

- leur désir de protéger l'enfant, mais cette attitude implique secret, suspicion, tabou ainsi que potentiellement une perte de confiance et

- la nécessité de parler à l'enfant qui est à la base de l'honnêteté et de la confiance dans la relation parent-enfant. Elle va engendrer la souffrance mais aussi rendre possible explications et soutien ainsi que l'émergence progressive chez l'enfant d'une stratégie de «vivre avec» personnelle.

Les parents doivent être soutenus dans leur recherche du moment où différer l'information devient plus préjudiciable que l'inévitable crise de la révélation. Un jeune qui ne pose pas de questions: est-ce parce qu'il ne veut pas savoir ou a-t-il perçu qu'il valait mieux protéger ses parents? Soutien, assistance professionnelle à longue échéance font partie du conseil génétique dans les situations difficiles. Dans ce contexte, les associations d'entraide jouent souvent un rôle très précieux et il est judicieux de toujours informer sur leur contribution possible.

Cela vaut la peine de parler aux enfants, de faire confiance à leur capacité de trouver avec le temps leur propre stratégie de vie à l'égard d'une situation difficile, comme chacun sait, un moment viendra inévitablement où il ne sera plus possible de protéger l'enfant de la vie réelle, mieux vaut alors qu'il y soit bien préparé. 